\title{
Pawlak's Conflict Model: Directions of Development
}

\author{
Alicja Wakulicz-Deja, Małgorzata Przybyła-Kasperek \\ University of Silesia \\ Institute of Computer Science \\ Będzińska 39, 41-200 Sosnowiec, Poland \\ Email: \{alicja.wakulicz-deja, malgorzata.przybyla-kasperek\}@us.edu.pl
}

\begin{abstract}
The article provides an overview of different approaches to the methods of conflict analysis that are inspired by the model of Zdzisław Pawlak. In the first part of the paper, Pawlak's original model is described. In the second part, the model proposed by Skowron and Deja is discussed. In the third part, the model proposed by the authors is presented.
\end{abstract}

\section{INTRODUCTION}

$\mathbf{I}$ $\mathrm{N}$ THE paper issues related to the problem of conflict analysis are considered. In 1984 Zdzisław Pawlak proposed a simple and intuitive model of conflict analysis. This model allows the relations between the units involved in the conflict to be defined and enables the conflict situation to be visualized and coalitions to be identified. In the model, the concept of agents was introduced and it is a multi-agent system, although it has nothing in common with the definition of the multi-agent systems that are known from the literature [9], [13], [37]. The first articles about this model were written by Pawlak and Skowron [18], [19], [20], [21], [22], [23], [24], [25], [38]. However, the intensive development of solutions to these problems occurred after Pawlak's death and was, in some sense, the realization of his intentions. The extensions of the model that were proposed allow for the analysis of conflicts in different situations as well as for numerous applications in real life situations. Some of these approaches stem from attempts to find solutions to real situations and the effects of different solutions to conflict. In this study, both the original conflict model of Pawlak as well as a review of the directions of its development are presented.

\section{PAWLAK'S CONFLICT MODEL}

Conflicts are inscribed in human nature and they have been with us since the dawn of history. Conflict analysis plays an important role in government, politics, business, lawsuits, disputes, negotiations, military operations, labor-management and others. In 1984, in the paper [18], a model to describe the complicated structure of conflict in a simple way was proposed by Zdzisław Pawlak. This issue was further developed by Pawlak in the papers [19], [20], [21], [22], [23], [24]. During the period in which Pawlak was interested in the issue of conflict, some other models of conflict situations were discussed in [1], [8], [10], [12], [15], [36]. Pawlak predicted that rough and fuzzy sets are the perfect candidates for modeling conflict situations in the presence of uncertainty, but at that time, not very much had been done in this area.

In the proposed model, the parties involved in the conflict are called agents. Depending on the type of conflict situation, that is considered, agents can be individuals, groups of individuals, institutions, companies, countries, etc. Each agent expresses his opinion about some discussed issue by assigning one of three values: -1 means, that an agent is against, 0 neutral toward the issue 1 means favorable. Knowledge about conflict situation is written in the form of table, in which rows are labeled by agents, and columns are labeled by discussed issues. The entries of the table are the values that were uniquely assigned to each agent and an issue. Each entry represents opinion of agent about issue. This type of table is an example of an information system $S=(U, A)$, that definition can be found in the literature [16], [17]. In the case of the Pawlak's conflict model elements of the universe $U$ are agents, $A$ is a set of issues, and the set of values of $a \in A$ is equal $V^{a}=\{-1,0,1\}$. The value $a(x)$, where $x \in U, a \in A$ is opinion of agent $x$ about issue $a$. For each $a \in A$ function $\phi_{a}: U \times U \rightarrow\{-1,0,1\}$ is defined:

$$
\phi_{a}(x, y)= \begin{cases}1 & \text { if } a(x) a(y)=1 \text { or } x=y \\ 0 & \text { if } a(x) a(y)=0 \text { and } x \neq y \\ -1 & \text { if } a(x) a(y)=-1\end{cases}
$$

Then over $U \times U$ three relations are defined: $R_{a}^{+}$alliance, $R_{a}^{0}$ neutrality, $R_{a}^{-}$conflict, that express the relations between agents:

$$
\begin{array}{ll}
R_{a}^{+}(x, y) & \text { if and only if } \phi_{a}(x, y)=1 \\
R_{a}^{0}(x, y) & \text { if and only if } \phi_{a}(x, y)=0, \\
R_{a}^{-}(x, y) & \text { if and only if } \phi_{a}(x, y)=-1
\end{array}
$$

Directly from the definition indicates that the alliance relation is

- reflexive: $\forall_{x \in U} R_{a}^{+}(x, x)$

- symmetric: $\forall_{x, y \in U}\left(R_{a}^{+}(x, y) \Rightarrow R_{a}^{+}(y, x)\right)$,

- transitive: $\forall_{x, y, z \in U}\left(R_{a}^{+}(x, y) \wedge R_{a}^{+}(y, z) \Rightarrow R_{a}^{+}(x, z)\right)$.

Relation $R_{a}^{+}$is an equivalence relation. Each equivalence class of alliance relation $R_{a}^{+}$is called coalition on $a$.

In order to evaluate views between agents $x$ and $y$ with respect to the set of issues $B \subseteq A$ a function of distance 
between agents $\rho_{B}^{*}: U \times U \rightarrow[0,1]$ is defined

$$
\rho_{B}^{*}(x, y)=\frac{\sum_{a \in B} \phi_{a}^{*}(x, y)}{\operatorname{card}\{B\}},
$$

where

$$
\begin{gathered}
\phi_{a}^{*}(x, y)=\frac{1-\phi_{a}(x, y)}{2}= \\
= \begin{cases}0 & \text { if } a(x) a(y)=1 \text { or } x=y, \\
0.5 & \text { if } a(x) a(y)=0 \text { and } x \neq y, \\
1 & \text { if } a(x) a(y)=-1 .\end{cases}
\end{gathered}
$$

In the definition of the function of distance between agents it is assumed that distance between agents that are in conflict is greater than distance between agents which are neutral. The function of distance between agents for the set of all issues $B=A$ is written in short as $\rho$.

Now we can in a more general way than before, without reference to specific issues, define the relations between agents. A pair $x, y \in U$ is said to be:

- allied $R^{+}(x, y)$, if $\rho(x, y)<0.5$,

- in conflict $R^{-}(x, y)$, if $\rho(x, y)>0.5$,

- neutral $R^{0}(x, y)$, if $\rho(x, y)=0.5$.

Set $X \subseteq U$ is a coalition if for every $x, y \in X, R^{+}(x, y)$ and $x \neq y$. Coalitions defined in this way does not have to be pairwise disjoint, which was shown in Example 2.1.

Each agent has the strength, that is expressed in the form of non-negative real number $\mu: U \rightarrow[0, \infty)$. The strength $\mu(x)$ of agent $x$ can represent economic or military power of a given agent. Each agent distributes his forces against his enemies according to a chosen strategy and knowledge of the situation. A strategy is defined as a function $\lambda: U \times U \rightarrow[0, \infty)$ that assigns a non-negative real number to each pair of agents $x, y$. This number expresses how much strength the agent $x$ directed against the agent $y$. It is reasonable to assume that for every $x, y$ :

$$
\begin{gathered}
\text { if } \rho(x, y) \leq 0.5 \text { then } \lambda(x, y)=0, \\
\sum_{y \in E_{x}} \lambda(x, y) \leq \mu(x),
\end{gathered}
$$

where $E_{x}$ is the set of all enemies of $x$, i.e., $E_{x}=\{y \in$ $U: \rho(x, y)>0.5\}$. The first condition indicates that the strength directed by $x$ against agents who are allied with $x$ or neutral to $x$ is zero. The second condition ensures that the sum of all the forces led by the $x$ against their enemies may not exceed the strength of agent $x$. Of course, the choice of strategy is essential in the case, which agents will win in a conflict situation, and which will lose.

Pawlak has defined a particularly important strategy, in which each agent has enough strength to destroy all of his enemies. A strategy of intimidation is a strategy $\lambda$ that fulfills the conditions:

$$
\begin{gathered}
\forall_{x \in U} \quad \sum_{y \in E_{x}} \lambda(x, y)=\mu(x), \\
\forall_{x, y \in U} \lambda(x, y)=\lambda(y, x) .
\end{gathered}
$$

The strategy of intimidation is unfavorable for all of the agents involved in the conflict, since its realization will cause that all agents will destroy each other.

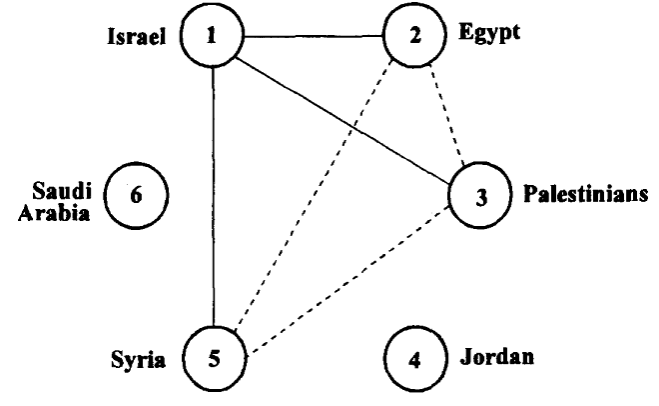

Fig. 1. A graphical representation of the Middle East conflict, issue $a$

Example 2.1: We consider an example named the Middle East conflict that is from the paper [22]. In the example there are six agents $U=\{1,2,3,4,5,6\}$

1 - Israel,

2 - Egypt,

3 - Palestinians,

4 - Jordan,

5 - Syria,

6 - Saudi Arabia

and five issues $A=\{a, b, c, d, e\}$

a - autonomous Palestinian state on the West Bank and Gaza,

b - Israeli military outpost along the Jordan River,

c - Israeli retains East Jerusalem,

d - Israeli military outposts on the Golan Heights,

e - Arab countries grant citizenship to Palestinians who choose to remain within their borders.

The relationship of each agent to a specific issue is presented in Table I.

As can be seen Egypt, Palestinian and Syria are allied on issue $a$, Jordan and Saudi Arabia are neutral to this issue whereas, Israel and Egypt, Israel and Palestinian, and Israel and Syria are in conflict about this issue. This can be easily illustrated by a graph. Figure 1 shows a graphical representation of the conflict situation. Agents are represented by circles in the figure. When a pair of agents is in conflict about the issue $a$, the circles representing the agents are linked. When agents are allied no this issue, the circles representing the agents are connected by dotted line.

The value of the function of the distance between agents is calculated for each pair of agents. These values are given in Table II. Now a graphical representation of a conflict situation, that takes into account all issues being considered by agents, is presented in Figure 2. As before when a pair of agents is in conflict, the circles representing the agents are linked. When agents are allied, the circles representing the agents are connected by dotted line. Neutral agents are not present in this conflict situation. In order to find coalitions, all cliques should be identified in the graph. So the subset of vertices such that every two vertices are connected by dotted line is determined. There are two coalitions in the Middle East conflict $\{1,6\}$ and $\{2,3,4,5\}$. 
TABLE I

INFORMATION SYSTEM FOR THE MIDDLE EAST CONFLICT

\begin{tabular}{c|ccccc}
$\mathrm{U}$ & $\mathrm{a}$ & $\mathrm{b}$ & $\mathrm{c}$ & $\mathrm{d}$ & $\mathrm{e}$ \\
\hline 1 & -1 & +1 & +1 & +1 & +1 \\
2 & +1 & 0 & -1 & -1 & -1 \\
3 & +1 & -1 & -1 & -1 & 0 \\
4 & 0 & -1 & -1 & 0 & -1 \\
5 & +1 & -1 & -1 & -1 & -1 \\
6 & 0 & +1 & -1 & 0 & +1
\end{tabular}

TABLE II

VALUES OF THE DISTANCE FUNCTION BETWEEN AGENTS

\begin{tabular}{l|llllll} 
& 1 & 2 & 3 & 4 & 5 & 6 \\
\hline 1 & & & & & & \\
2 & 0.9 & & & & & \\
3 & 0.9 & 0.2 & & & & \\
4 & 0.8 & 0.3 & 0.3 & & & \\
5 & 1.0 & 0.1 & 0.1 & 0.2 & & \\
6 & 0.4 & 0.5 & 0.5 & 0.4 & 0.6 &
\end{tabular}

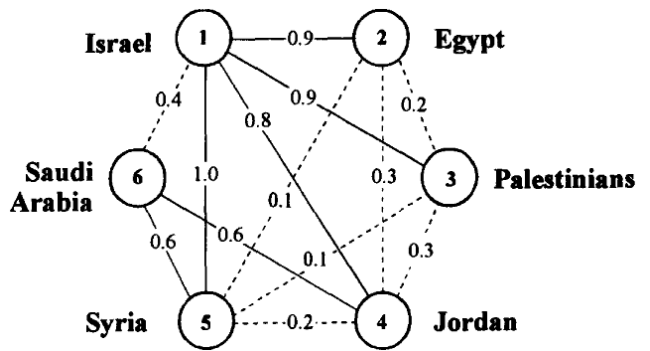

Fig. 2. A graphical representation of the Middle East conflict, distance between agents

The model of conflict analysis that was proposed by Pawlak allows to make advanced analysis of relations between agents and provides guidance that help to decide about strategy. Presented mathematical model is a simple way to illustrate the basic properties of conflicts.

The concepts described above were pursued by many authors [2], [3], [4], [5], [6], [7], [11], [14], [32], [33], [34], [35], [38], [39], [44], [45], [46], [47], [48], [49], [50], [51]. Some of these proposals are briefly described below.

\section{CONFLiCT MODEL PROPOSED BY SKOWRON AND DEJA}

Around 1996, Skowron and Deja proposed conflict model [2], which is an enhancement of the Pawlak model. This concept is described in the papers [3], [4], [5], [6], [38], [7]. Motivation to propose a new model was that, as the authors noted, in the Pawlak model a set of the attribute's values may be too limited in many real situations. Moreover, the assumption that the issues the agents vote represent the issues each agent takes care of causing that the model can be applied in very few real situations. In addition, the reason for the conflict cannot be determined because views on the issues to vote are consequences of the decision taken, based on the local issues, the current state and some background knowledge that are the real cause of the conflict. The main aim of a new model is to define and analyse causes of conflict. It was assumed that the conflict between agents is the consequence of the limited resources which are available to agents in a situation. If the number of resources is insufficient to attain agents' goals it often comes into the conflicts. In the proposed model, the fact was taken into consideration that any agent is describing the situation in its own way. The manner of understanding the same world by each agent can be completely different. A reflection of this assumption in the mathematical model is that for each agent a separate information system is assigned. It was assumed that the sets of attributes of different agents are pairwise disjoint.

Let $A g$ be a set of agents involved in conflict. Knowledge about the views of agent $a g \in A g$ on conflict situation is represented in an information system $S_{a g}=\left(U_{a g}, A_{a g}\right)$, where $U_{a g}$ - is a set of local states and $A_{a g}$ - is a set of attributes. It was assumed that the sets of attributes of different agents are pairwise disjoint

$$
\forall_{a g, a g^{\prime} \in A g ; a g \neq a g^{\prime}} \quad A_{a g} \cap A_{a g^{\prime}}=\emptyset .
$$

For each information system a target function $e_{a g}: U_{a g} \rightarrow$ $[0,1]$, that determines a subjective evaluation score to each state, is defined. The target function is used to determine the subset of local states, which are accepted by the agent with a fixed threshold value. A set of target states is $T_{a g}=\{s \in$ $\left.U_{a g}: e_{a g}(s)>\mu_{a g}\right\}$, where $\mu_{a g}$ is a level of acceptance, chosen subjectively by the agent $a g$. The set of target states in this model are presented in the form of Boolean formula.

Conflict is described by a situation and constraints. A situation $S$ is any element of the Cartesian product $S_{A g}=\prod_{a g \in A g} U_{a g}^{*}$, where $U_{a g}^{*}$ is a set of all possible local states of the agent $a g$. Constraints come from the bound on the number of resources and describe some dependencies among local states of agents. Constraints restrict the set of possible situations to admissible situations and are described by Boolean formulas.

The situations are evaluated. The score assigned to each situation can reflect the agents preferences (subjective states 
evaluation) or the expert judgement - who takes into account the global good. In the second evaluation method, a quality function $q: S_{A g} \rightarrow[0,1]$ is defined. The set of situations satisfying a given level of quality $t$ is defined by

$$
\text { Score }_{A g}(t)=\left\{S \in S_{A g}: q(S) \geq t\right\} .
$$

Boolean formula that describes the set $\operatorname{Score}_{A g}(t)$ is determined. Assessment of the situation expressed by preferences of the agents is defined by the global preference function $p: S_{A g} \rightarrow R, p(S)=F\left(e_{a g_{1}}\left(s_{1}\right), \ldots, e_{a g_{n}}\left(s_{n}\right)\right)$, where $S=\left(s_{1}, \ldots, s_{n}\right)$ and $n=\operatorname{card}\{A g\}$. Aggregation function $F$ may be chosen in many different ways. Boolean formula that describes the set of all preferred situations is also determined.

The multi-agent system, with local states for each agent defined and the global situations satisfying constraints, will be called the system with constraints and is denoted by $M_{A g}$. In such systems, conflict can be defined on several levels. Three types of conflicts are distinguished

- local conflict - that arises from the low level of subjective evaluation of the current state,

- global conflict (based on an expert evaluation) - indicates the existence of a situation which is not preferable for the global good,

- global conflict (based on agents preferences) - indicates that the current situation is not preferred by most of the agents.

The concepts of conflict, that are defined above, are the basis for investigation of the most fundamental problem - the possibility to achieve the consensus. In this model, the solution of conflict is searched on various levels: local and global, subjective and objective. In all cases, this is accomplished by determining the conjunction of Boolean formulas that describe appropriate conditions.

\section{CONFliCT MODEL PROPOSED BY WAKULICZ-DEJA AND PRZYBYŁA-KASPEREK}

Around 2009, Wakulicz-Deja and Przybyła-Kasperek proposed conflict model, which is also an extension of the Pawlak model. This concept is described in the papers [26], [27], [28], [29], [30], [31], [40], [41], [42], [43]. Motivation to propose a new model was that, the authors wanted to use conflict model for making decisions based on dispersed knowledge that is stored in many local decision tables. These tables are given in advance and collected by different units for example in different medical centers. It is not assumed that the sets of conditional attributes or the universe of different local decision tables are disjoint or equal. In the situation that is described above the Pawlak model can not be directly applied, because we are dealing with a set of decision tables - not just one information system. The Skowron - Deja model can not be applied, because in order to resolve conflicts the Boolean reasoning is used there and the algorithm has exponential pessimistic execution time. The identification of constraints in the considered dispersed situation is not possible. In addition, the assumption that the sets of conditional attributes of all decision tables must be disjoint is not fulfilled.
The main assumptions that were adopted in the proposed model are that knowledge is stored in several decision tables. There are a set of resource agents, one agent has access to one decision table. The resource agents, that are similar, in some specified sense, are combined into a group. In the process of groups creating elements of conflict analysis and negotiation are used. System has a hierarchical structure. For each group of agents a superordinate agent is defined - a synthesis agent. The synthesis agent has access to knowledge that is the result of the process of inference that is carried out by the resource agents that belong to its subordinate group. Based on local decisions taken by synthesis agents, global decisions are generated using certain fusion methods and methods of conflict analysis.

Different approaches to creating a system's structure were proposed: from a very simple solution to a more complex method of creating groups of agents. Below, a brief overview of the proposed approaches was presented.

The first approach was proposed in the papers [40], [41], [42]. In these articles definitions of resource agents and synthesis agents are given, and the hierarchical structure of the system was established. We call $a g$ in $A g$ a resource agent if it has access to the resources that are represented by a decision table $D_{a g}:=\left(U_{a g}, A_{a g}, d_{a g}\right)$, where $U_{a g}$ is ,the universe"; $A_{a g}$ is a set of conditional attributes, and $V_{a g}^{a}$ is a set of attribute $a$ values that contain the special signs $*$ and ?. The equation $a(x)=*$ for some $x \in U_{a g}$ means that for an object $x$, the value of attribute $a$ has no influence on the value of the decision attribute, while the equation $a(x)=$ ? means that the value of attribute $a$ for object $x$ is unknown; $d_{a g}$ is referred to as a decision attribute and $V_{a g}^{d}$ is called the value set of $d_{a g}$. The only condition that must be satisfied by decision tables of agents is the occurrence of the same decision attributes in all of the decision tables of the agents. In this first approach it was assumed that resource agents taking decisions on the basis of common conditional attributes form a group of agents called a cluster. For each cluster that contains at least two resource agents, a superordinate agent is defined, which is called a synthesis agent as. By a dispersed system we mean

$$
W S D_{A g}=\left\langle A g,\left\{D_{a g}: a g \in A g\right\}, A s, \delta\right\rangle,
$$

where $A g$ is a finite set of resource agents; $\left\{D_{a g}: a g \in A g\right\}$ is a set of decision tables of resource agents; $A s$ is a finite set of synthesis agents, $\delta: A s \rightarrow 2^{A g}$ is a injective function which each synthesis agent assign a cluster. A significant problem that must be solved when making decisions based on dispersed knowledge has identified - inconsistencies of knowledge may occur within the clusters. This problem stems from the fact that there are no assumptions about the relation between the sets of the conditional attributes of different resource agents in the system. We understand an inconsistency of knowledge to be a situation in which conflicting decisions are made on the basis of two different decision tables that have common conditional attributes and for the same values for the common attributes using logical implications. The process of generating the common knowledge (an aggregated decision table) for each cluster was proposed that was named as the process for the elimina- 
tion of inconsistencies in the knowledge. This method consists in constructing new objects that are based on the relevant objects from the decision tables of the resource agents that belong to one cluster. The aggregated objects are created by combining only those relevant objects for which the values of the decision attribute and common conditional attributes are equal. Based on the aggregated decision tables the values of decisions with the highest support of synthesis agents are selected. Global decisions are taken using the DBSCAN algorithm.

The second approach was proposed in the papers [26], [27], [43]. The same methods as in the first approach are used, however, additionally the decision tables of the resource agents are subjected to a certain transformation. In the paper [43], the method of editing and condensing were used on the decision tables of the resource agents. In the paper [26], based on the decision tables of the resource agents decision rules, using rough set theory, are generated. Then, in a single cluster, these rules are aggregated. In the article [27], from each decision tables of the resource agents unnecessary attributes are removed.

The third approach was proposed in the papers [28], [30]. The main modification, compared to the first approach, was conducted within the process of creating a system's structure. In the previous approaches a dispersed system has a static structure (created once, for all test objects), and this time a dynamic structure is used (created separately for each test object). The aim of this approach is to identify homogeneous groups of resource agents. The agents who agree on the classification into the decision classes for a test object will be combined in a group. The modified definitions of relations of friendship and conflict as well as the method for determining the intensity of the conflict, which were introduced by Pawlak, are used. Relations between agents are defined by their views for the classification of the test object to the decision classes. In the first step of the process of clusters creating for each resource agent $a g_{i}$ a vector of probabilities that reflects the classification of the test object is generated. Then, based on this vector, the vector of ranks $\left[r_{i, 1}(x), \ldots, r_{i, c}(x)\right]$, where $c=\operatorname{card}\left\{V^{d}\right\}$ is generated. We define the function $\phi_{v_{j}}^{x}$ for the test object $x$ and each value of the decision attribute $v_{j} \in V^{d}$; $\phi_{v_{j}}^{x}: A g \times A g \rightarrow\{0,1\}$

$$
\phi_{v_{j}}^{x}\left(a g_{i}, a g_{k}\right)= \begin{cases}0 & \text { if } r_{i, j}(x)=r_{k, j}(x) \\ 1 & \text { if } r_{i, j}(x) \neq r_{k, j}(x)\end{cases}
$$

where $a g_{i}, a g_{k} \in A g$.

We also define the intensity of conflict between agents using a function of the distance between agents. We define the distance between agents $\rho^{x}$ for the test object $x: \rho^{x}$ : $A g \times A g \rightarrow[0,1]$

$$
\rho^{x}\left(a g_{i}, a g_{k}\right)=\frac{\sum_{v_{j} \in V^{d}} \phi_{v_{j}}^{x}\left(a g_{i}, a g_{k}\right)}{\operatorname{card}\left\{V^{d}\right\}},
$$

where $a g_{i}, a g_{k} \in A g$.

We say that agents $a g_{i}, a g_{k} \in A g$ are in a friendship relation due to the object $x$, which is written $R^{+}\left(a g_{i}, a g_{k}\right)$, if and only if $\rho^{x}\left(a g_{i}, a g_{k}\right)<0.5$. Agents $a g_{i}, a g_{k} \in A g$ are in a conflict relation due to the object $x$, which is written $R^{-}\left(a g_{i}, a g_{k}\right)$, if and only if $\rho^{x}\left(a g_{i}, a g_{k}\right) \geq 0.5$.

Then, the groups of agents who are in agreement about the classification to the decision classes of the test object are defined. Two different approaches to combining agents in the friendship relations into one cluster were considered.

In the paper [30] the approach is proposed in which disjoint clusters of resource agents remaining in the friendship relations are created. The process of clusters creating in this approach is very similar to the hierarchical agglomerate clustering method and proceeds as follows. Initially, each resource agent is treated as a separate cluster. These two steps are performed until the stop condition, which is given in the first step, is met.

1) One pair of different clusters is selected (in the very first step a pair of different resource agents) for which the distance reaches a minimum value. If the selected value of the distance is less than 0.5 , then agents from the selected pair of clusters are combined into one new cluster. Otherwise, the clustering process is terminated.

2) After defining a new cluster, the value of the distance between the clusters are recalculated. The following method for recalculating the value of the distance is used. Let $\rho^{x}: 2^{A g} \times 2^{A g} \rightarrow[0,1]$, let $D_{i}$ be a cluster formed from the merger of two clusters $D_{i}=D_{i, 1} \cup D_{i, 2}$ and let it be given a cluster $D_{j}$ then

$$
\rho^{x}\left(D_{i}, D_{j}\right)=
$$

$$
\begin{cases}\frac{\rho^{x}\left(D_{i, 1}, D_{j}\right)+\rho^{x}\left(D_{i, 2}, D_{j}\right)}{2}, & \text { if } \rho^{x}\left(D_{i, 1}, D_{j}\right)<0.5 \\ & \text { and } \rho^{x}\left(D_{i, 2}, D_{j}\right)<0.5 \\ \max \left\{\rho^{x}\left(D_{i, 1}, D_{j}\right), \rho^{x}\left(D_{i, 2}, D_{j}\right)\right\}, & \text { if } \rho^{x}\left(D_{i, 1}, D_{j}\right) \geq 0.5 \\ & \text { or } \rho^{x}\left(D_{i, 2}, D_{j}\right) \geq 0.5\end{cases}
$$

In the paper [28] the approach is proposed in which not disjoint clusters are created. The cluster due to the classification of object $x$ is the maximum, due to the inclusion relation, subset of resource agents $X \subseteq A g$ such that $\forall a g_{i}, a g_{k} \in X \quad R^{+}\left(a g_{i}, a g_{k}\right)$. Thus, the cluster is the maximum, due to inclusion relation, set of resource agents that remain in the friendship relation due to the object $x$.

In both approaches, the same definition of dispersed system is used. By a dispersed decision-making system with dynamically generated clusters, we mean

$$
W S D_{A g}^{d y n}=\left\langle A g,\left\{D_{a g}: a g \in A g\right\},\right.
$$

$\left\{A s_{x}: x\right.$ is a classified object $\},\left\{\delta_{x}: x\right.$ is a classified object $\left.\}\right\rangle$ where $A g$ is a finite set of resource agents; $\left\{D_{a g}: a g \in A g\right\}$ is a set of decision tables of the resource agents; $A s_{x}$ is a finite set of synthesis agents defined for the clusters that are dynamically generated for test object $x, \delta_{x}: A s_{x} \rightarrow 2^{A g}$ is an injective function that each synthesis agent assigns to the cluster that is generated due to classification of object $x$.

Also in both approaches the method of elimination of inconsistencies in the knowledge and the DBSCAN algorithm, that were proposed in the first approach, are used.

The fourth approach was proposed in the paper [29]. In this approach a dynamic structure is also used, but the process 
of clusters creating is more extensive and as a consequence the clusters are more complex and better reconstructed and illustrate the views of the agents on the classification. The main differences between this approach and the previous approach are as follows. Now, three types of relations between agents are defined: friendship, neutrality and conflict (previously, only two types were used). The clustering process consists of two stages (previously, only one stage process was used). In the first step initial groups are created, it contains agents in friendship relation. In the second stage, a negotiation stage, agents which are in neutrality relation are attached to the existing groups. In order to define the intensity of conflict between agents two function are used: the distance function between agents (was used in the previous approach) and a generalized distance function. The process of clusters creating is as follows. At first the distance between agents $\rho^{x}$ is defined. Definitions of the relations between agents are modeled on the definitions that were given by Pawlak. Let $p$ be a real number that belongs to the interval $[0,0.5)$. We say that agents $a g_{i}, a g_{k} \in A g$ are in a friendship relation due to the object $x$, which is written $R^{+}\left(a g_{i}, a g_{k}\right)$, if and only if $\rho^{x}\left(a g_{i}, a g_{k}\right)<0.5-p$. Agents $a g_{i}, a g_{k} \in A g$ are in a conflict relation due to the object $x$, which is written $R^{-}\left(a g_{i}, a g_{k}\right)$, if and only if $\rho^{x}\left(a g_{i}, a g_{k}\right)>0.5+p$. Agents $a g_{i}, a g_{k} \in A g$ are in a neutrality relation due to the object $x$, which is written $R^{0}\left(a g_{i}, a g_{k}\right)$, if and only if $0.5-p \leq \rho^{x}\left(a g_{i}, a g_{k}\right) \leq 0.5+p$. The first step in the process of creating clusters is to define the initial cluster. The initial cluster due to the classification of object $x$ is the maximum, due to the inclusion relation, subset of resource agents $X \subseteq A g$ such that $\forall_{a g_{i}, a g_{k} \in X} R^{+}\left(a g_{i}, a g_{k}\right)$. After the first stage of clustering we obtain a set of initial clusters and a set of agents which are not included in any cluster. In this second group of agents there are agents which remained undecided. So those which are in neutrality relation with agents belonging to some initial clusters. In the second step, the negotiation stage, this agents play a key role. As it is known the goal of negotiation process is to reach a compromise by accepting some concessions by the parties involved in a conflict situation. In the negotiation process, the intensity of the conflict is determined by using the generalized distance function. This definition assumes that during the negotiation, agents put the greatest emphasis on compatibility of ranks assigned to the decisions with the highest ranks. That is the decisions that are most significant for the agent. Compatibility of ranks assigned to less meaningful decision is omitted. We define the function $\phi_{G}^{x}$ for test object $x$; $\phi_{G}^{x}: A g \times A g \rightarrow[0, \infty)$

$$
\phi_{G}^{x}\left(a g_{i}, a g_{j}\right)=\frac{\sum_{v_{l} \in \operatorname{Sign}_{i, j}}\left|r_{i, l}(x)-r_{j, l}(x)\right|}{\operatorname{card}\left\{\operatorname{Sign}_{i, j}\right\}}
$$

where $a g_{i}, a g_{j} \in A g$ and $\operatorname{Sign}_{i, j} \subseteq V^{d}$ is the set of significant decision values for the pair of agents $a g_{i}, a g_{j}$. We also define the generalized distance between agents $\rho_{G}^{x}$ for the test object $x ; \rho_{G}^{x}: 2^{A g} \times 2^{A g} \rightarrow[0, \infty)$

$$
\rho_{G}^{x}(X, Y)=
$$

$$
\left\{\begin{array}{cc}
0 & \text { if } \quad \operatorname{card}\{X \cup Y\} \leq 1 \\
\frac{\sum_{a g, a g^{\prime} \in X \cup Y} \phi_{G}^{x}\left(a g, a g^{\prime}\right)}{\operatorname{card}\{X \cup Y\} \cdot(\operatorname{card}\{X \cup Y\}-1)} & \text { else }
\end{array}\right.
$$

where $X, Y \subseteq A g$. As can be easily seen, the value of the generalized distance function for two sets of agents $X$ and $Y$ is equal to the average value of the function $\phi_{G}^{x}$ for each pair of agents $a g, a g^{\prime}$ that belong to the set $X \cup Y$. For each agent which is not attached to any cluster we calculate the value of generalized distance function for this agent and every initial cluster. Then the agent is included to all initial clusters, for which the generalized distance does not exceed a certain threshold, which is set by the system's user. Also agents without coalition, for which the value does not exceed the threshold, are combined into a new cluster. We do not connect agents who are in conflict relation in one cluster. After completion of the second stage of the process of clustering we get the final form of clusters. Then the method of elimination of inconsistencies in the knowledge and the DBSCAN algorithm, that were proposed in the first approach, are used.

The fifth approach was proposed in the paper [31]. In this paper, a method of creating clusters is the same as in the previous approach but when the global decisions are taken the agents' weights are additionally calculated. Different methods of calculating the strength of a cluster was proposed:

- with respect to the number of component agents,

- with respect to the decisiveness of agents,

- with respect to the number of component agents and the decisiveness of agents,

- with respect to the decisiveness-based cluster strength.

Further work is carried out on the development of a dispersed system. The authors propose another approach in order to achieve a better efficiency of the system and always find inspiration in the work of Pawlak. The relations as well as the method for determining the intensity of a conflict between agents that were proposed by Pawlak are always the basis of the entire system.

\section{SUMMARY}

In this article, the conflict analysis model that was proposed by Pawlak has been described. The aim of the study was to show the main extensions of this model that have been proposed in the literature. Pawlak's model is simple, intuitive and very useful in the analysis of the complex nature of conflicts. The model has many applications and is inspiration for developing new approaches.

\section{REFERENCES}

[1] Coombs, C.H.,Avruin, G.S.: The Structure of Conflicts. Lawrence Erlbaum, London, 1988.

[2] Deja, R.: Conflict analysis. in: S. Tsumoto, S. Kobayashi, T. Yokomori, H. Tanaka, A. Nakamura, (Eds.), Proceedings of the Fourth International Workshop on Rough Sets, Fuzzy Sets and Machine Discovery, The University of Tokyo, 6-8 November, (1996) 118-124. 
[3] Deja, R.: Conflict Model with Negotiation. Bulletin of the Polish Academy of Sciences, Technical Sciences, 44 (4), (1996) 475-498.

4] Deja, R.: Conflict analysis. Proceedings of the 7th European Congress on Intelligent Techniques \& Soft Computing, Aachen, Germany, September 13-16, 1999.

[5] Deja, R.: Conflict Analysis. Rough Set Methods and Applications; New Developments. In: L. Polkowski, et al. (eds.), Studies in Fuzziness and Soft Computing, Physica-Verlag, (2000) 491-520.

[6] Deja, R.: Application of rough set theory in conflict analysis. Instytu Podstaw Informatyki Polskiej Akademii Nauk, Dissertation, supervisor: A. Skowron 2000.

[7] Deja, R.: Conflict analysis, Int. J. Intell. Syst., 17, 2, (2002) 235-253, http://dx.doi.org/10.1002/int.10019

[8] Dorclan, P.: Interaction under conditions of crisis: Application of graph theory to internation relations. Peace Research Society International Papers 11 (1969) 89-109.

[9] Franklin, S., Graesser, A.: Is it an Agent, or just a Program - A Taxonomy for Autonomous Agents, Proceedings of the Third International Workshop on Agent Theories, Architectures, and Languages, Berlin: Springer-Verlag, (1996) 21-35

[10] Hart, H.: Structures of influence and cooperation-conflict. International Interactions 1 (1974) 141-162.

[11] Kanczewski, A.: On regular conflicts. Bull. Polish Acad. Sci. Math. 33 (11/12) (1985) 685-692.

[12] Maeda, Y., Senoo, K., Tanaka, H. Interval density function in conflict analysis. in: N. Zhong, A. Skowron, S. Ohsuga (Eds.), New Directions in Rough Sets, Data Mining and Granular-Soft Computing, Springer, New York, (1999) 382-389.

[13] Maes, P.: Artificial Life Meets Entertainment: Life like Autonomous Agents, Communications of the ACM (38) (1995) 108-114

[14] Nabialek, I.: Convex sets of balenced strategies in conflict situations. Bull. Polish Acad. Sci. Math. 36 (1988) 425-428.

[15] Nakamura, A.: Conflict Logic with Degrees. in: S.K. Pal, A. Skowron (Eds.), Rough Fuzzy Hybridization-A New Trend in Decison-Making, Springer, New York, (1999) 136-150.

[16] Pawlak, Z.: Information Systems. Theoretical foundation. WNT, Warszawa (1983) (in polish)

[17] Pawlak, Z.: Rough Sets: Theoretical aspects of reasoning about data Kluwer Academic Publishers, Boston, 1991.

[18] Pawlak, Z.: On conflicts. Int. J. of Man-Machine Studies 21, (1984) 127-134.

[19] Pawlak, Z.: About conflicts (in Polish), Polish Scientic Publishers, Warsaw, (1987) 1-72.

[20] Pawlak, Z.: Anatomy of conflict. Bulletin of the European Association for Theoretical Computer Science, 50, (1993) 234-247.

[21] Pawlak, Z: On some issues connected with conflict analysis. Institute of Computer Science Reports, 37/93, Warsaw University of Technology 1993.

[22] Pawlak, Z.: An Inquiry Anatomy of Conflicts. Journal of Information Sciences 109, (1998) 65-78.

[23] Pawlak, Z.: Some remarks on conflict analysis. European Journal of Operational Research 166, (2005) 649-654, http://dx.doi.org/10.1016/j.ejor.2003.09.038

[24] Pawlak, Z.: Conflicts and Negotations. In: Wang, G.-Y., Peters, J.F., Skowron, A., Yao, Y. (eds.) RSKT 2006. LNCS (LNAI), vol. 4062, Springer, Heidelberg (2006) 12-27, http://dx.doi.org/10.1007/11795131_2

[25] Pawlak, Z., Skowron, A.: Rough Sets and Conflict Analysis. E-Service Intelligence: Methodologies, Technologies and Applications, (2007) 3574, http://dx.doi.org/10.1007/978-3-540-37017-8_2

[26] Przybyła-Kasperek M., Wakulicz-Deja A.: Application of decision rules, generated on the basis of local knowledge bases, in the process of global decision-making, Intelligent Decision Technologies Smart Innovation, Systems and Technologies, Vol. 1, Part 2, (2012) 375-388

[27] Przybyła-Kasperek M., Wakulicz-Deja A.: Application of reduction of the set of conditional attributes in the process of global decision-making, Fundamenta Informaticae 122 (4), (2013) 327-355, http://dx.doi.org/10.3233/FI-2013-793

[28] Przybyła-Kasperek M., Wakulicz-Deja A.: Global decision-making system with dynamically generated clusters, Information Sciences Volume 270, (2014) 172-191, http://dx.doi.org/10.1016/j.ins.2014.02.076

[29] Przybyła-Kasperek M., Wakulicz-Deja A.: A dispersed decision-making system - The use of negotiations during the dynamic generation of system's structure, Information Sciences, Volume 288, (2014) 194-219, http://dx.doi.org/10.1016/j.ins.2014.07.032

[30] Przybyła-Kasperek M., Wakulicz-Deja A.: Global decision-making in multi-agent decision-making system with dynamically generated disjoint clusters, Applied Soft Computing, 40, (2016) 603-615, http://dx.doi.org/10.1016/j.asoc.2015.12.016

[31] Przybyła-Kasperek M., Wakulicz-Deja A.: The strength of coalition in a dispersed decision support system with negotiations, European Journal of Operational Research, 252, (2016) 947-968, http://dx.doi.org/10.1016/j.ejor.2016.02.008

[32] Ramanna, S., Peters, J.F., Skowron, A.: Generalized Conflict and Resolution Model with Approximation Spaces. Rough Sets and Current Trends in Computing, 5th International Conference, RSCTC 2006 , Kobe, Japan, November 6-8, 2006, Proceedings, (2006) 274-283, http://dx.doi.org/10.1007/11908029_30

[33] Ramanna, S., Peters, J.F., Skowron, A.: Approaches to Conflict Dynamics Based on Rough Sets. Fundam. Inform. 75(1-4) (2007) 453-468.

[34] Ramanna, S., Skowron, A.: Requiremnents Interaction an Conflicts A Rough Set Approach. Proceedings of the IEEE Symposium on Foundations of Computational Intelligence, FOCI 2007, part of the IEEE Symposium Series on Computational Intelligence 2007, Honolulu, Hawaii, USA, 1-5 April 2007, (2007) 308-313, http://dx.doi.org/10.1109/FOCI.2007.372185

[35] Ramanna, S., Skowron, A., Peters, J.F.: Approximation SpaceBased Socio-Technical Conflict Model. Rough Sets and Knowledge Technology, Second International Conference, RSKT 2007, Toronto, Canada, May 14-16, 2007, Proceedings (2007) 476-483, http://dx.doi.org/10.1007/978-3-540-72458-2_59

[36] Roberts, F.: Discrete Mathematical Models with Applications to Social, Biological and Environmental Problems, Prentice-Hall, Englewood Cliffs, NJ, 1976.

[37] Russell, S., Norvig, P.: Artificial Intelligence: A Modern Approach, Prentice Hall, Englewood Cliffs, New Jersey, 1995.

[38] Skowron, A., Deja, R.: On Some Conflict Models and Conflict Resolutions. Romanian Journal of Information Science and Technology 3(1-2), (2002) 69-82.

[39] Skowron, A., Ramanna, S. Peters, J.F. Conflict Analysis and Information Systems: A Rough Set Approach. Rough Sets and Knowledge Technology, First International Conference, RSKT 2006 , Chongqing, China, July 24-26, 2006, Proceedings, (2006) 233-240, http://dx.doi.org/10.1007/11795131_34

[40] Wakulicz-Deja A., Przybyła-Kasperek M.: Hierarchical Multi-Agent System, Recent Advances in Intelligent Information Systems, Academic Publishing House EXIT, (2009) 615-628.

[41] Wakulicz-Deja A., Przybyła-Kasperek M.: Global decisions Taking on the Basis of Multi-Agent System with a Hierarchical Structure and Density-Based Algorithm, CS\&P, Uniwersytet Warszawski, (2009) 616627.

[42] Wakulicz-Deja A., Przybyła-Kasperek M.: Multi-Agent Decision Taking System, Fundamenta Informaticae 101(1-2), (2010) 125-141, http://dx.doi.org/10.3233/FI-2010-280

[43] Wakulicz-Deja A., Przybyła-Kasperek M.: Application of the method of editing and condensing in the process of global decision-making, Fundamenta Informaticae 106 (1), (2011) 93-117, http://dx.doi.org/10.3233/FI2011-378

[44] Wąsowski, J.: Existence of the balanced Strategy in theory of conflicts. Bull. Polish Acad. Sci. Math. 35 (1987) 535-537.

[45] Wiweger, A.: On the notation of a conflict, Bull. Polish Acad. Sci. Math $34(5 / 6)(1986) 381-391$.

[46] Xuat, N.V.: Security in the theory of conflicts. Bull. Polish Acad. Sci. Math. 32 (1984) 539-541.

[47] Żakowski, W.: On new characterization of regular configurations in theory of conflict situations, Demonstration Mathematica 17 (1984) 211218.

[48] Żakowski, W.: Investigation of balanced situation in theory of conflicts, Bull. Polish Acad. Sci. Math. 32 (7/8) (1984) 379-382.

[49] Żakowski, W.: The balanced state in a total-conflict situation, Bull. Polish Acad. Sci. Math. 33 (7/8) (1985) 379-382.

[50] Żakowski, W.: On some properties of sets of configurations in theory of conflicts, Bull. Polish Acad. Sci. Math. 34 (1986) 123-126.

[51] Żakowski, W.: The balanced strategy in an oriented total-conflict, Bull. Polish Acad. Sci. Math. 35 (1987) 525-530. 